\title{
Study on Remodulation Scheme in WDM-PON Utilizing a Reflective Semiconductor Optical Amplifier
}

\author{
Kejun YANG ${ }^{1, a}$, Wei HONG ${ }^{2, b}$ \\ ${ }^{1}$ Wuhan National Laboratory for Optoelectronics, Huazhong University of Science and Technology, \\ Wuhan 430074, China; \\ ${ }^{2}$ Wuhan National Laboratory for Optoelectronics, Huazhong University of Science and Technology, \\ Wuhan 430074, China. \\ ayangkj0331@163.com,w.hong@hust.edu.cn
}

Keywords:remodulation;WDM-PON;semiconductor optical amplifier

Abstract. Influences of length, injected optical power, internal loss, differential gain coefficient and back facet reflectivity on remodulation in RSOA-based WDM-PON are investigated. The remodulation effect can be improved by optimizing structural, operating and physical parameters.

\section{Introduction}

Wavelength division multiplexed passive optical network (WDM-PON) is considered as the most promising candidate for the next generation technology to satisfy the ever increasing bandwidth demand [1]. Signal remodulation of the downstream signal to produce the upstream signal can reduce the cost owing to carrier reuse [2].Reflective semiconductor optical amplifier (RSOA) is one of the attractive solutions for colorless optical network unit (ONU) because it doesn't need any extra broadband light sources at the central office (CO) and can provide enough power budgets for the upstream signals due to its gain property [3].

In this paper,we focus on influences of structural, operating and physical parameters, i.e. length, injected optical power, internal loss, differential gain coefficient and reflectivity of back facet,on RSOA used for remodulation in WDM-PON.Since the influences of these parameters are greater than others,we choose them for study.To be spercific,we study the influences of the parameters mentioned above on the receiver sensitivity at bit error ratio(BER) of $1 \cdot 10-4$, which is the threshold of forward error correction(FEC). The result shows that the performance of RSOA can be effectively improved by optimizing structural, operating and physical parameters, which has not been discussed in literatures to the best of our knowledge.

\section{Simulation architecture}

The WDM-PON architecture considered in our simulation is shown in Fig.1.The inset shows the RSOA gain versus injected power, which is helpful to explain the gain saturation effect of SOA.

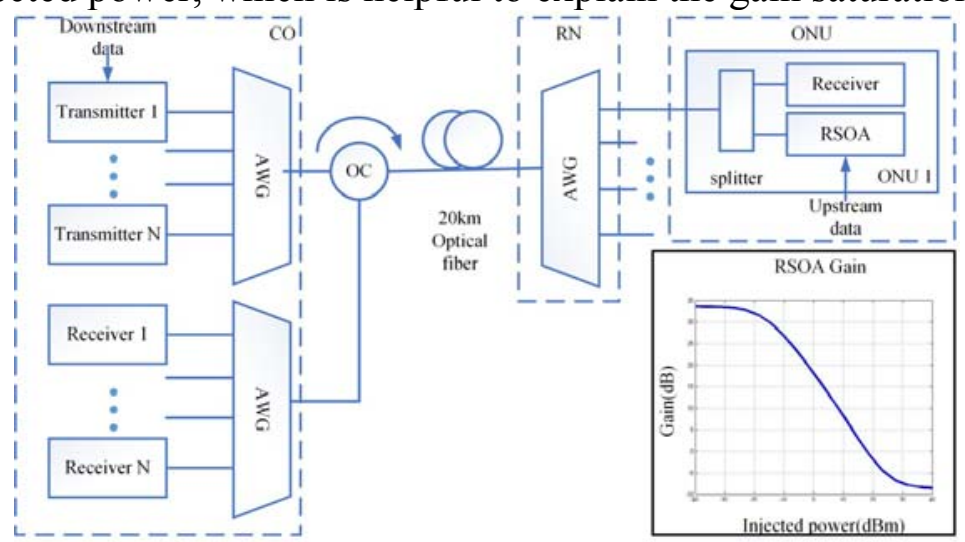

Fig. 1. WDM-PON architecture. (AWG: arrayed waveguide grating; OC: optical circulator; CO: central office; RN: remote node; ONU:optical network unit) 
In our simulation the wavelength of the transmitted light is $1550 \mathrm{~nm}$. It is modulated with a 10 $\mathrm{Gb} / \mathrm{s}$ NRZ signal of 128-bit pseudo-random data. The extinction ratio is set to $4 \mathrm{~dB}$. The signal is then transmitted through $20 \mathrm{~km}$ long optical fiber and then splitted by an asymmetric splitter in ONU. About 1/85 of the signal is sent to downstream receiver and the other part is injected into the RSOA. The splitting ratio is designed to satisfy the downstream power budget requirement. As depicted in the inset above, the gain of SOA is reduced for higher injected optical power as a result of gain saturation effect. This effect can be used to erase the downstream signal when light propagates through the RSOA. Meanwhile, the injection current of RSOA carrying $10 \mathrm{~Gb} / \mathrm{s}$ upstream signal remodulates the downstream light. Then the light is reflected back to the transmission fiber and received in the CO. However, if the RSOA is operated in saturation region, modulation extinction ratio will be reduced while parasitic chirp will be enlarged. As a result, upstream transmission performance will be degraded. Consequently, the operating parameters and even physical and structural parameters should be carefully designed to optimize the upstream transmission performance.

First we use MATLAB to build the model of RSOA which is described in [4],then we use the commercial software Optisystem to build a WDM-PON transmission system and put the RSOA model into the ONU to erase the downstream signal and remodulate the light with the upstream data.

\section{Simulation results and discussions}

First we consider the influence of the length of RSOA(Length) on the receiver sensitivity evaluated at bit error ratio (BER) of $1 \cdot 10^{-4}$ (the receiver sensitivity mentioned below is measured under the same condition). The result is shown in Fig.2.
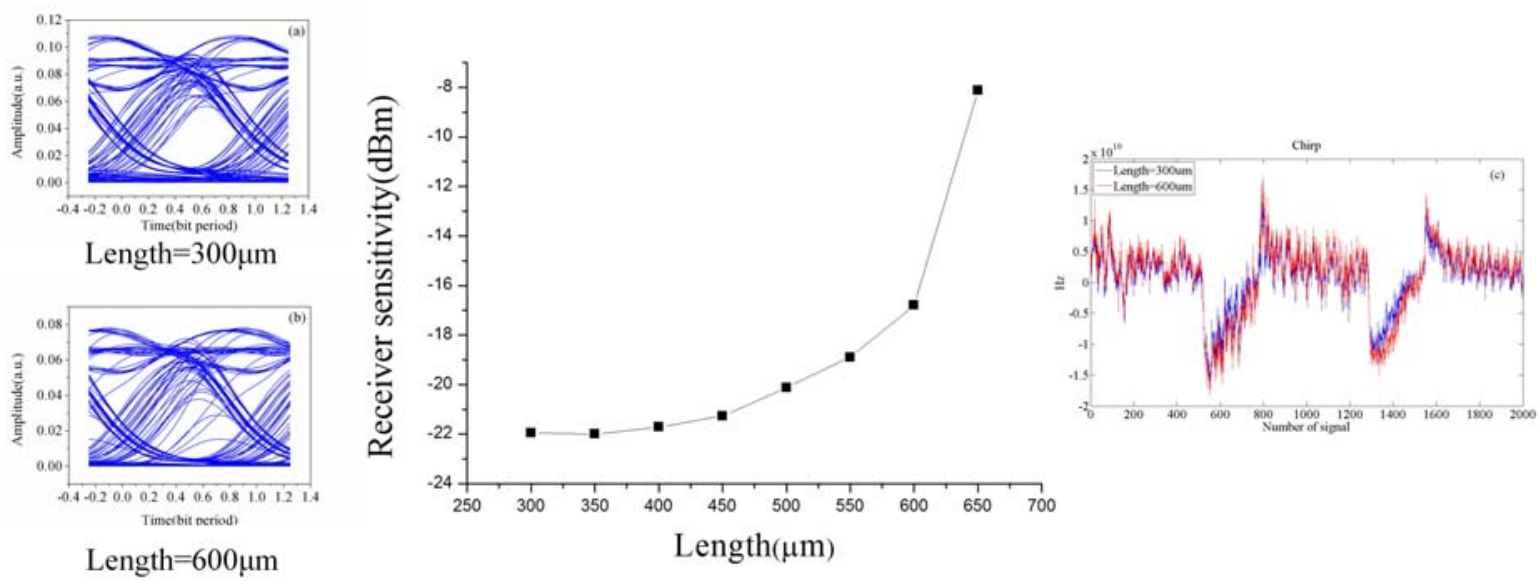

Fig.2.Receiver sensitivity versus length

From Fig.2. we can see that the receiver sensitivity decreases with the length of RSOA increasing,which means the quality of upstream signal deteriorates. This is because the shorter the cavity is, the deeper the degree is for RSOA to work in gain saturation region when the injected current is constant due to higher carrier density[5].As a result of this, the downstream signal is erased more clearly in shorter RSOA, which can be seen from the comparison of eye diagrams of different lengths above.From the insets above we can see that the interference from downstream to upstream signal in RSOA with shorter length is less than that in RSOA with longer length due to bigger opening of the eye.Moreover,with length of RSOA increasing (while injection current level is unchanged), the carrier density level decreases, and the extinction ratio of the upstream optical signal degrades, which degrades the receiver sensitivity. We can find the fact from the magnitude of "1" level in the inset figures (a) and (b) in Fig. 2. Meanwhile, with lower carrier density, the RSOA will be more saturated. As a result, the chirp of upstream signal generated by a longer RSOA is larger than that of a shorter RSOA, which can be seen from the inset figure (c). Chirp can significantly affect the signal quality after transmission through dispersion fiber. 

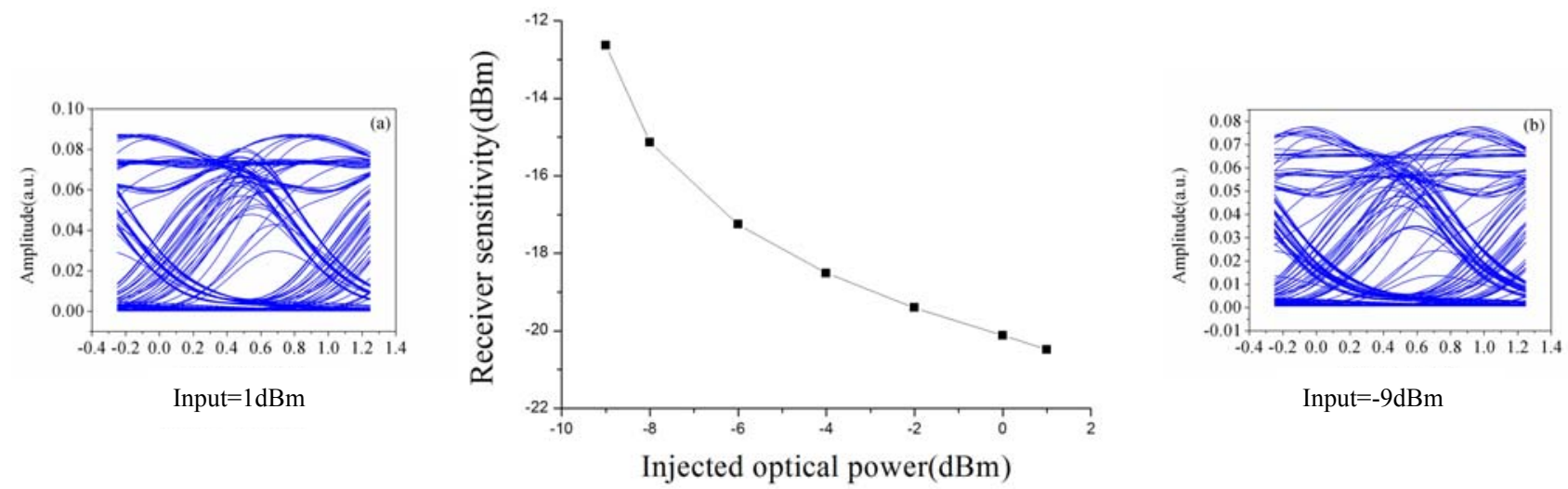

Fig.3.Receiver sensitivity versus injected optical power

Fig.3. shows receiver sensitivity as a fuction of injected optical power into the RSOA (Input). It is shown that receiver sensitivity increases with injected optical power increasing. Higher injected optical power causes severer gain saturation so that the downstream signal can be erased more clearly, leading to the improvement of upstream signal quality. We can see this effect from the comparison of " 1 " level thickness in the eye diagrams for different injection powers in inset figures (a) and (b). The insets clearly show that the trace of " 1 " level is narrower for higher injected optical power. Besides,signal to noise ratio(SNR) also affects the result. The calculated SNR for $1 \mathrm{dBm}$ and $-9 \mathrm{dBm}$ injected optical power is $45.6 \mathrm{~dB}$ and $32.5 \mathrm{~dB}$ respectively, which can be attributed to supression of amplified spontaneous emission(ASE) noise from the RSOA for higher input optical powers. As a result,the receiver sensitivity increases because of less influence of noise on receiving signal.
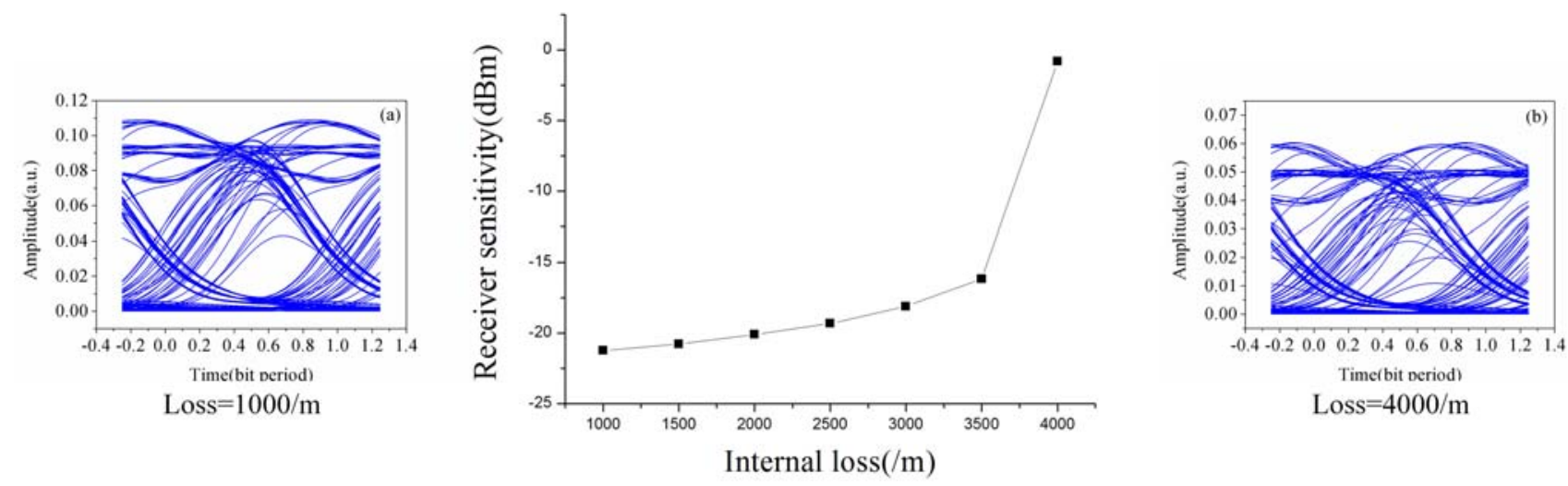

Fig.4.Receiver sensitivity versus internal loss

The relationship of receiver sensitivity versus internal loss(Loss) is shown in Fig.4.The eye diagrams with internal loss of $1000 / \mathrm{m}$ and $4000 / \mathrm{m}$ are shown in the nearby insets.As displayed in the figure above,receiver sensitivity witnesses a course of decrease with internal loss growing.The reason can be attributed to the effect of gain saturation.The RSOA with higher internal loss tends to work in the linear gain region due to lower optical power.The downstream signal is,therefore,erased less clearly.As a result,the quality of upstream signal converted from this downstream signal degrades due to more interaction between them. This result can be seen clearly from the eye diagrams above. The eye in the eye diagram of $4000 / \mathrm{m}$ loss opens narrower than that of $1000 / \mathrm{m}$ loss because of more interference from residual downstream signal. Another reason is the extinction ratio.It can also be seen from the comparison of eye diagrams above. 


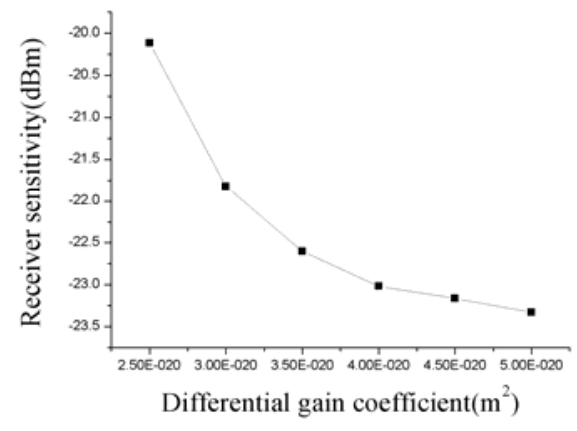

Fig.5.Receiver sensitivity versus differential gain coefficient

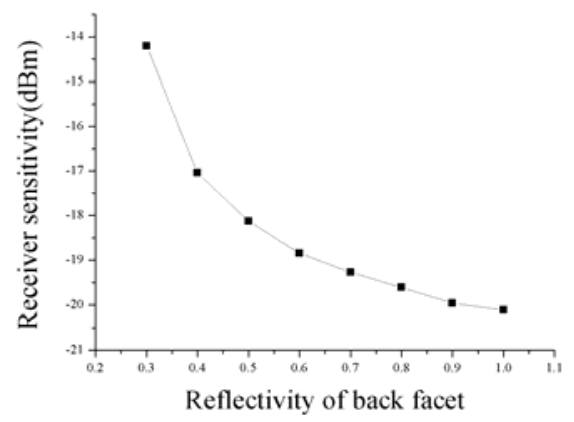

Fig.6.Receiver sensitivity versus reflectivity of back facet

Fig.5. and Fig.6. show receiver sensitivity as a function of differential gain coefficient and reflectivity of back facet, respectively. It is shown that receiver sensitivity increases when differential gain coefficient and reflectivity of back facet increase. Gain of RSOA is positively proportional to the differential gain coefficient, which is on the opposite of the effect of increasing internal loss. Higher reflectivity of back facet causes higher reflected optical power in RSOA, which can be thought as equivalent to increase of input optical power. The result of Fig.6. can be, therefore, attributed to the same effect as previously mentioned when explaining Fig. 3..

\section{Conclusion}

We investigate the relationships between uplink receiver sensitivity and length, injected optical power, internal loss ,differential gain coefficient and reflectivity of back facet, respectively. The results show that the erasure of downstream signal and the quality of remodulated upstream signal can be effectively improved by optimizing structural, operating and physical parameters of RSOA.

\section{References}

[1] C. H. Lee, W. V. Sorin, and B. Y. Kim, "Fiber to the Home Using a PON Infrastructure," IEEE J. Lightw. Technol., vol. 24, no.20, pp. 4568-4583, Dec. 2006

[2] Chow, C. W., et al. "Signal remodulation high split-ratio hybrid WDM-TDM PONs using RSOA-based ONUs." Electronics letters 45.17 (2009): 903-905.

[3] S. Y. Kim, S. B. Jun, Y. Takushima, E. S. Son, and Y. C. Chung, "Enhanced performance of RSOA-based WDM PON by using Manchester coding”, J. opt. net, Vol. 6, No. 6, pp.624-630, June 2007

[4] Connelly, Michael J. "Wideband semiconductor optical amplifier steady-state numerical model." IEEE Journal of Quantum Electronics,vol.37,no.3,pp. 439-447,March 2001 
[5] N. S. Ribeiro, A. L. R. Cavalcante, C. M. Gallep, and E. Conforti, "Data rewriting after carrier erasing by ultra-long SOA", in Proc.Opt.Fiber Commun.Conf.(OFC)/Natl.Fiber Optic

Eng.Conf.(NFOEC), JWA42 , 2011. 